\title{
A KALUZA-KLEIN PICTURE OF ELECTRIC MAGNETIC DUALITY
}

\section{IN SUPERSYMMETRY}

\author{
P. A. Zizzi
}

CERN - Geneva

The supersymmetry algebra for the (4+1)-dimensional $\mathrm{N}=2$ supersymmetric $\mathrm{SU}(2)$ Yang-Mills theory, in the phase of broken gauge symmetry, includes a surface integral $G_{\mu}$, which is a part of the momentum having a topological origin.

There are static non-trivial classical solutions, periodic on $x_{5}$, which contribute to $G_{5}$ in the gauge $A_{5}=0$.

$G_{5}$ gives rise, by dimensional reduction to $3+1$ dimensions, to the magnetic charge of the 't HooftPolyakov monopoles, which acquires an interpretation analogous to that of the electric charge in the Kaluza-Klein theory.

*)

On leave of absence from King's College, Department of Mathematics, Strand, London WC 2R 2LS, England. 
The electric-magnetic duality conjecture ${ }^{1)}$, restricted by Montonen and Olive ${ }^{2)}$ to the 't Hooft-Polyakov model ${ }^{3)}$, was strongly suggested by the universal mass formula which is valid, at the classical level, for both elementary particles and solitons ('t Hooft-Polyakov monopoles).

Witten and Olive ${ }^{4)}$ showed that the universal mass formula can survive quantization when the theory is taken supersymmetric, as the supersymmetry algebra includes both the electric charge and the magnetic charge of the 't Hoof't-Polyakov monopoles as central charges. In this context, Olive ${ }^{5)}$ interpreted the electric charge and the magnetic charge, respectively, as the fifth and sixth extra components of the four-momentum.

The topological origin of the magnetic charge in the higher dimensions becomes more evident when the intermediate step in five dimensions is taken into account, in the dimensional reduction from six to four dimensions. In fact, the supersymmetry algebra for the (4+1)-dimensional theory, in the phase of unbroken gauge symmetry, has a central charge ${ }^{6)}$ that is the topological charge of the fivedimensional solitons (instantons in the four-dimensional Euclidean space). This topological central charge gives rise, by dimensional reduction to $3+1$ dimensions, to the magnetic charge of the 't Hooft-Polyakov monopoles.

In this paper, we consider the (4+1)-dimensional theory in the phase of broken gauge symmetry. The supersymmetry algebra includes a surface integral, $G_{\mu}$, which is a part of the momentum having a topological origin. There are static non-trivial classical solutions periodic on $x_{5}$, which contribute to $G_{5}$, in the gauge $A_{5}=0$, and "collapse" to 't Hooft-Polyakov monopoles in the dimensional reduction from $4+1$ to $3+1$ dimensions, which resembles a spontaneous compactification ${ }^{7)}$.

By dimensional reduction to $3+1$ dimensions, $G_{5}$ gives rise to the magnetic charge of the 't Hooft-Polyakov monopoles, which acquires an interpretation, analogous to that of the electric charge in the Kaluza-Klein theory ${ }^{8)}$, of a translation generator on the fifth periodic dimension. $\left[G_{5}\right.$ is the generator of a $U(1)$ magnetic group.] By a redefinition of the Casimir operators of the Poincaré group in $4+1$ dimensions, we get a mass formula for the zero mode of the periodic topological configurations contributing to $G_{5}$ 。 
We connect our results in the phase of broken gauge symmetry of the theory, to the ones obtained in the phase of unbroken gauge symmetry ${ }^{61}$. We find that the (4+1)-dimensional supersymmetry algebra exhibits electric-magnetic duality between the two phases of the theory. This gives rise to a complete fivedimensional picture of electric-magnetic duality (and chiral invariance) in the (3+1)-dimensional supersymmetry algebra. Finally, we relate this five-dimensional picture to a six-dimensional one 5$), 9)$.

In Section 2, we give a short review of the supersymmetry algebra for the USp(2) supersymmetric SU(2) Yang-Mills theory in 4+1 dimensions, in the phase of unbroken gauge symmetry, taking into account the dimensional reduction to $3+1$ dimensions. In section 3 , we calculate the (4+1)-dimensional supersymmetry algebra in the phase of broken gauge symmetry, and perform the dimensional reduction to $3+1$ dimensions. In section 4 , we study the topological features of the (4+1)-dimensional theory in the phase of broken gauge symmetry in relation with the results of Section 3. In Section 5, we illustrate the dimensional reduction from $5+1$ to $4+1$ dimensions (in both the phases of the theory) and from $4+1$ to $3+1$ dimensions in terms of topological maps. In this context, we also study the breaking of the Poincare invariance of the supersymmetry algebra.

\section{2. - THE SUPERSYMMETRY ALGEBRA FOR THE (4+1)-DIMENSIONAL THEORY IN THE PHASE OF UNBROKEN GAUGE SYMMETRY}

Let us consider the USp(2) supersymmetric SU(2) Yang-Mills theory in $4+1$ dimensions on-shell ${ }^{6)}$. The fields in the Lagrangian density are : a vector field $A_{\mu}^{a}(\mu=0,1,2,3,5 ; a=1,2,3)$, a real scalar field $\phi^{a}$, and two four-component complex USp(2) Majorana spinors $x_{i}^{a}(i=1,2)$. All the fields are in the adjoint representation of the gauge group $\mathrm{SU}(2)$.

In the phase of unbroken gauge symmetry of the theory $(\langle\phi\rangle=0)$, the supersymmetry algebra is ${ }^{6)}$ :

$$
\begin{aligned}
& \left\{\bar{Q}_{\alpha}^{i}, Q_{\beta}^{j}\right\}=\Omega^{i j}\left[\left(\gamma_{\mu}\right)_{\beta \alpha} P^{\mu}+\delta_{\alpha \beta} Z_{T}\right] \\
& (\mu=0,1,2,3,5 ; \alpha, \beta=1,2,3,4 ; i, j=1,2)
\end{aligned}
$$


$-3-$

where $\Omega^{i j}$ is the symplectic matrix and

$$
Z_{T}=-\frac{1}{4} \int d^{4} \vec{x} F_{\ell m}^{a} \widetilde{F} \lim a
$$

is the topological charge of the five-dimensional solitons (instantons in the fourdimensional Euclidean space). The solitons have a mass :

$$
M=\left|Z_{T}\right|
$$

The mass formula (2.3) that holds at the classical level in absence of the Highs mechanism, survives quantization 10). We remark that (2.3) shows the lack of electric-magnetic duality in the (4+1)-dimensional supersymmetry algebra (2.1).

In the dimensional reduction of the supersymmetry algebra (2.1) to $3+1$ dimensions $\left(A_{5}^{a}=\phi^{a}, \partial_{5}=0\right)$ the fifth component of the momentum, and the topological central charge give, respectively, for $\left\langle\phi^{\prime}\right\rangle \neq 0$ 9):

$$
\begin{aligned}
& P_{5} \rightarrow L_{E}\left(\phi^{\prime}\right)=\frac{1}{\left\langle\phi^{\prime}\right\rangle} \int d^{3} \vec{x}^{\prime} \partial_{i}\left(\phi^{\prime} a \text { Foin } a\right) \\
& Z_{T} \rightarrow G\left(\phi^{\prime}\right)=\frac{1}{\left\langle\phi^{\prime}\right\rangle} \int d^{3} \vec{x}^{\prime} \varepsilon_{i j k} \partial_{i}\left(\phi^{\prime a} F j k a\right) \\
& (i, j, k=1,2,3)
\end{aligned}
$$

where $Z_{E}\left(\phi^{\prime}\right)$ in $(2.4)$ is the electric charge of the unbroken Abelian subgroup $U(1)$, and $G\left(\phi^{\prime}\right)$ is the magnetic charge of the 't Hooft-Polyakov monopoles.

The (3+1)-dimensional supersymmetry algebra, obtained by dimensional reduction from the (4+1)-dimensional one given in (2.1), includes both the electric charge (2.4) and the magnetic charge (2.5) as central charges, in phase $\left\langle\phi^{\prime}\right\rangle \neq 0$ $(\langle\phi\rangle=0)$ :

$$
\begin{aligned}
& \left\{Q_{i \alpha} \overline{Q_{j \beta}}\right\}=\delta_{i j}\left(\delta_{\mu}\right)_{\alpha \beta} P^{\mu}+\varepsilon_{i j}\left[\left(\gamma_{5}\right) Z_{\alpha \beta}\left(\phi^{\prime}\right)+\delta_{\alpha \beta} G\left(\phi^{i}\right)\right]_{(2.6)} \\
& (\mu=0,1,2,3 ; \alpha, \beta=1,2,3,4 ; i, j=1,2)
\end{aligned}
$$

and the mass formula

$$
M^{2}=\left\langle\phi^{\prime}\right\rangle^{2}\left[Z_{E}^{2}\left(\phi^{\prime}\right)+G^{2}\left(\phi^{\prime}\right)\right]
$$

exhibits electric-magnetic duality under $Z_{E}\left(\phi^{\prime}\right), G\left(\phi^{\prime}\right)$ rotations ${ }^{2)}$. 
Notice that the dimensional reduction $(2.5)$ shows that the magnetic charge has a topological origin in $4+1$ dimensions. This can also be illustrated in terms of topological maps. In fact, the map of the 't Hooft-Polyakov monopoles can be obtained, by dimensional reduction, from the map of instantons periodic on the fifth dimension ${ }^{9)}$.

\section{3. - THE (4+1)-DIMENSIONAL SUPERSYMMETRY ALGEBRA IN THE PHASE OF BROKEN GAUGE SYMMETRY}

In Section 2, we recovered electric-magnetic duality in the (3+1)-dimensional supersymmetry algebra (2.6), by dimensional reduction from the (4+1)-dimensional supersymmetry algebra (2.1), where electric-magnetic duality was absent.

At this stage, however, we have not yet recovered the complete result of witten and olive ${ }^{4)}$, by dimensional reduction from $4+1$ dimensions. In fact, in (2.6), the chiral invariance of the $(3+1)$-dimensional supersymmetry algebra, under the exchange $\phi^{\prime} \phi^{\prime}$, is missed $\left(A_{5}=\phi^{\prime}, A_{6}=\phi\right)$.

Then, we must get the supersymmetry algebra, that is the chiral transformed of the one given in $(2,6)$, by dimensional reduction from the $(4+1)$-dimensional supersymmetry algebra calculated in the phase of broken gauge symmetry $(\langle\phi\rangle \neq 0)$.

Let us explicitly calculate the supersymmetry algebra for the USp(2) supersymmetric SU(2) Yang-Mills theory in 4+1 dimensions, in the phase of broken gauge symmetry. The expression of the supersymmetry charges in terms of the fields and their conjugate momenta is given in Ref. 6).J Paying attention to boundary terms, we get :

$$
\begin{aligned}
& \left\{\bar{Q}_{\alpha}^{i}, Q_{\beta}^{j}\right\}=S^{i j}\left[\left(\delta_{\mu}\right)_{\beta \alpha}\left(p^{\mu}+G^{\mu}\right)+\delta_{\alpha \beta} L_{E}\right] \\
& (\mu=0,1,2,3,5 ; \alpha, \beta=1,2,3,4 ; i, j=1,2)
\end{aligned}
$$

In (3.1), the central charge $Z_{E}$, given by

$$
\begin{aligned}
& Z_{E}(\phi)=\frac{1}{\langle\phi\rangle} \int d^{4} \vec{x} \partial \rho\left(\phi^{a} F \text { ol } a\right) \\
& (1=1,2,3,5 ; a=1,2,3)
\end{aligned}
$$


$-5-$

is the electric charge of the unbroken Abelian subgroup $U(1)$, and $G_{\mu}$ is given by :

$$
G_{\mu}=\frac{1}{\langle\phi\rangle} \int d^{4} \vec{x} R_{O \mu}
$$

where $R_{o \mu}$ is the time-component of the antisymmetric tensor:

$$
R_{\nu \mu}=\varepsilon_{\nu \mu \rho \sigma \tau} F \rho \sigma a\left(D^{\tau} \phi\right)^{a}
$$

$R_{V \mu}$ in (3.4) is the total divergence of a totally antisymmetric tensor $I_{\rho \nu \mu}$ :

$$
R_{\nu \mu}=\partial^{\rho} I_{\rho \nu \mu}
$$

where (by the use of the Bianchi identities) :

$$
I_{\rho \nu \mu}=\varepsilon_{\rho \nu \mu \sigma \tau} \phi^{a} F^{\sigma \tau a}
$$

and $R_{v \mu}$ is automatically conserved :

$$
\partial^{\nu} R_{\nu \mu}=0
$$

Then, $G_{\mu}$ in $(3.3)$ is the integral of a spatial divergence:

$$
G_{\mu}=\frac{1}{\langle\phi\rangle} \int d^{4} \vec{x} \partial^{\rho}\left(\varepsilon_{\rho \sigma \mu \sigma \tau} \phi^{a} F^{\sigma \tau a}\right)
$$

with $G_{0} \equiv 0$.

Notice that $G_{\mu}$ is not a vector under $0(4,1)$ rotations. Following Olive ${ }^{5)}$ who obtained an analogous result as $R_{v \mu}$ in $(3.4)$ in $5+1$ dimensions, we take $\mathrm{R}_{v \mu}$ as the antisymmetric part of the energy-momentum tensor $\theta_{v \mu}$.

Then, $\left(P_{\mu}+G_{\mu}\right)$ which is gauge invariant and conserved, is the true vector under $0(4,1)$ :

$$
\left[M_{\mu \nu},\left(P_{\rho}+G_{\rho}\right)\right]=-i\left[\eta_{\rho \mu}\left(P_{\nu}+G_{\nu}\right)-\eta_{\rho \nu}\left(P_{\mu}+G_{\mu}\right)\right]_{(3.8)}
$$

and it must be considered as the generator of translations in $4+1$ dimensions. 
In the following, we will show that $G_{\mu}$ in (3.7) has a topological origin, and does not vanish at infinity. Then, we will interpret $G_{\mu}$ as a part of the momentum which has non-zero eigenvalues only on soliton states. In the Hilbert subspace corresponding to elementary particle states $\left(G_{\mu}=0\right)$, we recover the usual definition for the momentum.

Let us perform the dimensional reduction of the supersymmetry algebra (3.1) to 3+l dimensions. The fifth component of the momentum, the electric charge in (3.2) and the fifth component of $G_{\mu}$ in (3.7), give, respectively, for $\left\langle\phi^{\prime}\right\rangle=0$

$$
\begin{aligned}
& P_{5} \rightarrow 0 \\
& Z_{E}(\phi) \rightarrow \hat{Z}_{E}(\phi)=\frac{1}{\langle\phi\rangle} \int d^{3} \vec{x} \partial_{i}\left(\phi^{a} F \text { oi } a\right) \\
& G_{5}(\phi) \rightarrow G(\phi)=\frac{1}{\langle\phi\rangle} \int d^{3} \vec{x} \varepsilon_{i j k} \partial_{i}\left(\phi^{a} F^{j k a}\right)
\end{aligned}
$$

$$
(i, j, k=1,2,3 ; a=1,2,3)
$$

The $(3+1)$-dimensional supersymmetry algebra obtained by dimensional reduction from (3.1) includes both the electric charge $Z_{E}(\phi)$ in (3.9) and the magnetic charge $G(\phi)$ in (3.10) as central charges.

$$
\begin{aligned}
& \left\{Q_{i \alpha} \bar{Q}_{j \beta}\right\}=\delta_{i j}\left(X_{\mu}\right){ }_{\alpha \beta}{ }^{\mu}+\varepsilon_{i j}\left[\delta_{\alpha \beta} \hat{L}_{E}(\phi)+\left(\gamma_{5}\right)_{\alpha \beta} G(\phi)\right] \\
& (\mu=0,1,2,3 ; \alpha, \beta=1,2,3,4 ; i, j=1,2)
\end{aligned}
$$

The mass formula

$$
M^{2}=\langle\phi\rangle^{2}\left[\hat{Z}_{E}^{2}(\phi)+G^{2}(\phi)\right]
$$

exhibits electric-magnetic duality under $\hat{Z}_{E}(\phi), G(\phi)$ rotations.

The supersymmetry algebra (3.11) is the chiral transformed of the one given in (2.6), under the exchange $\phi^{\prime} \phi^{\prime}$, and our result is now complete.

Notice that if the surface integral $G_{5}$ should vanish at infinity, we would miss the magnetic charge in the $(3+1)$-dimensional supersymmetry algebra (3.11), with a consequent lack of electric-magnetic duality, violation of the mass formula (3.12) and breaking of supersymmetry. 


\section{4. - TOPOLOGICAL FEATURES OF THE (4+1)-DIMENSIONAL THEORY IN THE PHASE OF BROKEN GAUGE SYMMETRY}

In Section 3 we showed that only the fifth component, $G_{5}$, of the "vectorial magnetic charge" in (3.7) (in the axial gauge $A_{5}^{a}=0$ ) plays a relevant rôle in the dimensional reduction to $3+1$ dimensions, giving rise to the magnetic charge of the 't Hooft-Polyakov monopoles $G(\phi)$ in (3.10).

In this section, we wish to enlighten the topological origin in $4+1 \mathrm{di-}$ mensions of $G(\phi)$. Thus, we will look for topological configurations in the $(4+1)-$ dimensional theory, in the phase of broken gauge symmetry, which contribute to $G_{5}$, in the axial gauge $A_{5}^{a}=0$.

We started by considering the theory defined in the five-dimensional Minkowski space $R_{M}^{5}$, with metric $\eta_{\mu \nu}=(+,-\cdots),(\mu, \nu=0,1,2,3,5)$. Now, let us take all the fields

$$
\bigoplus^{a}\left(x_{\mu}\right)=\left(A_{\mu}^{a}\left(x_{\mu}\right), \phi_{\left(x_{\mu}\right)}^{a}, X_{i}^{a}\left(x_{\mu}\right)\right)
$$

periodic on $x_{5}$

$$
\prod_{\mu^{\prime}=0,1,2,3}^{a}\left(x_{\mu^{\prime}}, x_{5}+R\right)=\prod_{0 \leq x_{5} \leq R}^{a}\left(x_{\mu}^{\prime}, x_{5}\right)
$$

The periodic condition (4.1), which is consistent with the metric, is equivalent to assume that the fifth dimension is a circle $S^{1}$ of radius $R$. The topology of our space is then

$$
R_{M}^{4} \times S^{1}
$$

where $R_{M}^{4}$ is the four-dimensional Minkowski space and $S^{1}$ is the periodic fifth dimension. Let us Fourier expand all the fields $\Phi^{a}\left(x_{\mu}\right)$ as

$$
\Phi^{a}\left(x_{\mu^{\prime}}, x_{5}\right)=\frac{1}{R^{1 / 2}} \sum_{n=-\infty}^{n=+\infty} \Phi_{(n)}^{a}\left(x_{\mu}^{\prime}\right) e^{i n \frac{2 \pi}{R} x_{5}}
$$

As we are looking for static classical solutions, we will restrict our space $(4.2)$ to 
$-8-$

$R_{E}^{3} \times S^{1}$

$(4.4)$

where $R_{E}^{3}$ is the three-dimensional Euclidean space. The static classical solustrons will be field configurations of the kind

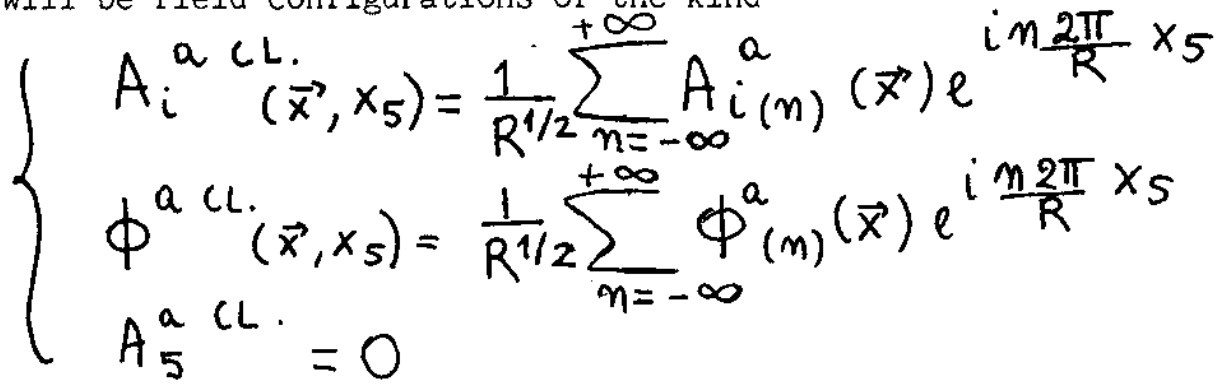

$$
\begin{aligned}
& \underline{\underline{i}}=1,2,3 ; \vec{x} \equiv\left(x_{1}, x_{2}, x_{3}\right)_{3} \text { (The coupling constant } g \text { has been rescaled } \\
& \text { to } \left.g^{1}=R^{\frac{3}{2}} g_{0}\right)
\end{aligned}
$$

defined on $R_{E}^{3} \times S^{1}$. The field configurations (4.5) are required to be topolotical maps :

$$
S^{2} \times S^{1} \longrightarrow \frac{S U(2)}{U(1)}
$$

where $S^{2}$ in $(4.6)$ is the boundary of $R_{E}^{3}$ :

$$
S^{2}=\left\{\left(x_{1}, x_{2}, x_{3}\right) \in R_{E}^{3} \mid \sum_{i=1}^{3} x_{i}^{2}=1\right\}
$$

and $\operatorname{SU}(2) / U(\lambda) \simeq S^{2}$. Then, we will consider the maps:

$$
\psi: S^{2} \times S^{1} \longrightarrow S^{2}
$$

The maps (4.8) are classified by the cohomotopy group :

$$
T^{2}\left(S^{2} \times S^{1}\right)=\mathbb{Z}
$$

Indeed, the homotopy classes of maps $\left[S^{2} \times S^{1}, S^{2}\right]$ can be proved to correspond to the cohomology group $\left.\mathrm{H}^{2}\left(\mathrm{~S}^{2} \times S^{1}\right)=\mathbb{Z} 11\right)$. Notice that the topological confgurations which are maps $\psi$ in (4.8), have a "hyper-toroidal" symmetry $S^{2} \times S^{1}$, and the radius of the "hypertorus" is the radius $R$ of the periodic fifth dimention. Among all the maps $\psi$ in (4.8), let us now consider the projection

$$
\pi:\left(\vec{x}, x_{5}\right) \longrightarrow \vec{x} \in S^{2}
$$


The choice of the projection $\pi$ restricts our topological configurations to the ones which do not depend on $x_{5}$. This corresponds to take the limit $R \rightarrow 0$ in the dimensional-reduction procedure.

In this case, the maps (4.10) "collapse" to the maps of the 't HooftPolyakov monopoles:

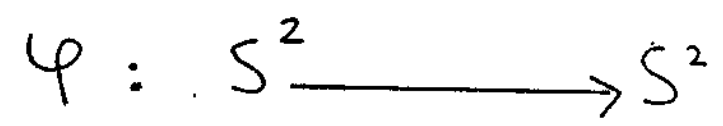

classified by the homotopy group :

$$
\Pi_{2}\left(S^{2}\right)=\mathbb{Z}
$$

as can also be seen in the trivial closure of the following diagram of spheres :

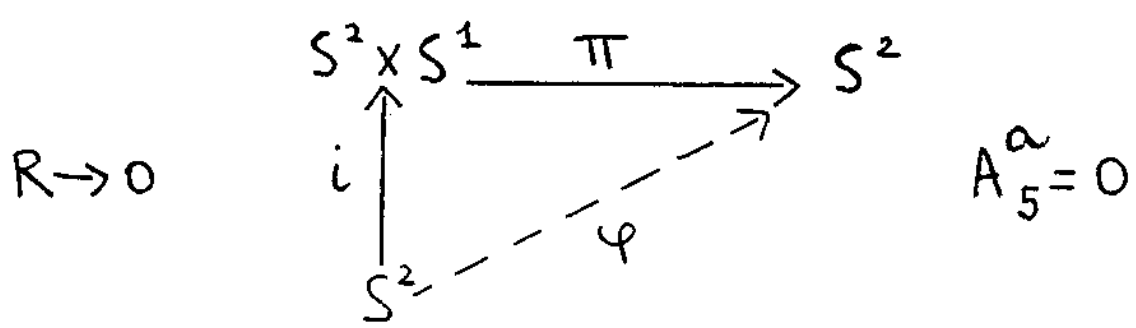

which illustrates the dimensional reduction from $4+1$ to $3+1$ dimensions in a purely geometrical way, $i_{0} e .$, in absence of Higgs fields $\left(A_{5}^{a}=0\right)$.

Then, the limit $R \rightarrow 0$ in the dimensional reduction procedure can be viewed as a cut of the "hypertorus" $S^{2} \times S^{1}$ through $S^{1}$ the embedding $i$ in the diagram (4.12)]. This cut gives rise to a 't Hooft-Polyakov monopole, with finite size, in $\mathrm{R}_{\mathrm{E}}^{3}$. For this reason, in this paper we will call the topological configurations which are maps (4.10) "pre-monopoles".

Let us consider now the limit $R \rightarrow \infty$. In this limit, the periodicity on $x_{5}$ is lost and we recover the original theory defined in $R^{5} M^{\circ}$

The (static) topological configurations that are to be considered now are the maps

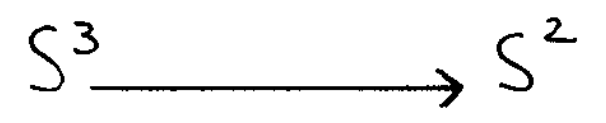

classified by the homotopy group : $\prod_{3}\left(S^{2}\right)=\mathbb{Z}$ 
$-10-$

where $S^{3}$ is the boundary of $R_{E}^{4}$ :

$$
S^{3}=\left\{\left(x_{1}, x_{2}, x_{3}, x_{5}\right) \in R^{4} E \mid \sum_{i} x_{i}^{2}=1\right\}
$$

and $S^{2} \simeq S U(2) / U(1)$. In particular, we will be interested in topological confgurations with topological number 1 . So, in the corresponding class of maps (4.13), we can choose the Hop map $\left.h: S^{3} \rightarrow S^{2} 12\right)$. Notice that the topological configurations which are maps (4.13) do not contribute to $G_{5}$. Hop solitons have been considered by de Vega in the three-dimensional compactified Euclidean space 13). Scaling arguments (Derrick theorem 14), show that topological configurations, which are maps (4.13), do not minimize the action in $4+1$ dimensions.

Instead, the topological configurations, periodic on $x_{5}$, which are maps (4.8) and contribute to $G_{5}$ in the gauge $A_{5}=0$, are minima for the action as is shown by the following arguments.

The Casimir operators of the Poincare group in $4+1$ dimensions are ${ }^{10)}$ :

$$
\begin{aligned}
& c_{1}=P_{\mu}^{\prime 2} \\
& c_{2}=\frac{1}{4} W_{\mu \nu} W^{\mu \nu} \\
& c_{3}=\frac{1}{8 M} \varepsilon_{\mu \nu \rho \sigma \tau} W^{\mu \nu} W^{\rho \sigma} p^{\prime \tau}
\end{aligned}
$$

where $\mu, \nu, \rho, \delta, \tau=0,1,2,3,5, W_{\mu \nu}$ is the generalization of the Pauli-Lubansky vector

$$
W_{\mu \nu}=\frac{1}{2 M} \varepsilon_{\mu \nu \rho \sigma \tau} M^{\rho \sigma} P^{\prime} \tau
$$

and in (4.15) $P_{\mu}^{\prime}$ is defined as :

$$
P_{\mu}^{\prime}=P_{\mu}+G_{\mu}
$$

For elementary particle states $\left(G_{\mu}=0\right)$ we have, as usual :

$$
P_{\mu}{ }^{2}=c_{1}
$$


For soliton states $\left(G_{\mu} \neq 0\right.$ for $\left.\mu=5, A_{5}=0\right)$, we have:

$$
\left(P_{\mu}+G_{\mu}\right)^{2}=C_{1}
$$

where we have taken $G_{\mu}=\left(0,0,0,0, G_{5}\right)$.

By dimensional reduction to $3+1$ dimensions, $G_{5}$ gives rise to the magnetic charge of the 't Hooft-Polyakov monopoles $G$, given in (3.10) and $P_{5}$ gives identically zero. From (4.19), we get, by dimensional reduction to $3+1$ dimensions :

$$
\begin{aligned}
& P \mu^{2}=G^{2}+C_{1} \\
& (\mu=0,1,2,3)
\end{aligned}
$$

In the rest frame : $P_{\mu}=(M, \vec{O})$, it is :

$$
M_{\text {monopole }}^{2}=G^{2}+C_{1}
$$

The mass formula for the monopoles is satisfied for $c_{1}=0$. Thus, in $(4.19) c_{1}$ is fixed to be zero by the dimensional reduction to $3+1$ dimensions. Equation (4.19) in the rest frame $P_{\mu}=(M, ठ),(\mu=0,1,2,3,5)$, is then

$$
\mathrm{M}_{\text {pre-monopole }}^{2}=G_{5}^{2}
$$

which is the mass formula for the pre-monopoles $(4.8)$.

Then, the topological configurations periodic on $x_{5}$ are minima for the action, while the non-periodic topological configurations are not. This means that the periodic condition (4.1) is not put "by hand" into the dimensional reduction procedure, but is required by the classical solutions with non-trivial. topology. In the limit $R \rightarrow 0$, the reduced action (where we take only the $n=0$ mode, which keeps a finite mass) is minimized by the 't Hooft-polyakov monopole solutions. This resembles a spontaneous compactification 7 ).

Notice that there is only one pre-monopole mode that is of physical interest, the zero mode, which satisfies the mass formula (4.22). All the other modes can be interpreted as excitations of monopoles, which have no physical meaning in $4+1$ dimensions. 
The periodic condition (4.1) breaks the Poincare invariance of the $(4+1)$-dimensional supersymmetry algebra (2.1) (in phase $\langle\phi\rangle=0$ ) and (3.1) (in phase $\langle\phi\rangle \neq 0$ ), respectively, as

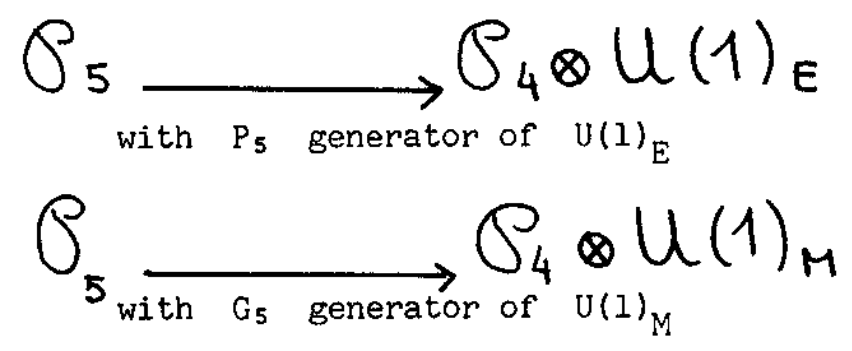

As $P_{5}$ gives rise to the electric charge $Z_{E}\left(\phi^{\prime}\right)$ in the dimensional reduction (2.4) to $3+1$ dimensions, $U(1)_{E}$ in (4.23) is the "electric" $U(1)$ group. In fact, Olive ${ }^{5)}$ showed that the picture of the electric charge in the Kaluza-Klein theory (no Higgs fields, periodicity on $x_{5}$ ) is equivalent to the Higgs field picture where the Higgs field $\left(\phi^{a^{\prime}}=A_{5}{ }^{a}\right)$ is gauged away $\left(A_{5}^{a}=0\right)$ by a gauge transformation which is periodic on $x_{5}$ in vacuo. Moreover, we will call $U(1)_{M}$ in (4.24) the "magnetic" $U(1)$ group [the dual group of $U(1)_{E}$ in (4.23)]. In this case, the magnetic charge $G(\phi)$ in $(3.10)$ plays a rôle similar to the electric charge in the Kaluza-Klein theory of a translation generator on the fifth periodic dimension.

The (4+1)-dimensional supersymmetry algebra exhibits electric-magnetic duality between the two phases of the theory :

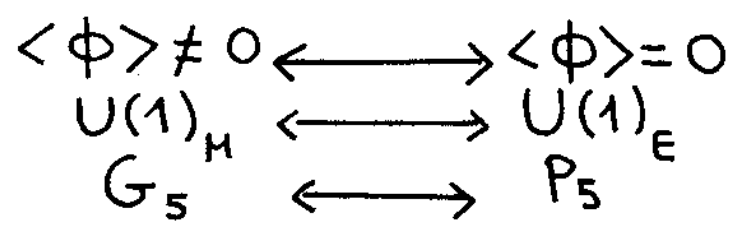

with an electric central charge $Z_{E}(\phi)$ in phase $\langle\phi\rangle \neq 0$ and a topological central charge $Z_{T}$ in phase $\langle\phi\rangle=0$. The central charges $Z_{E}$ and $Z_{T}$ are reminiscent of the generators of an "electric" $U(1)$ group and a "magnetic" $U(1)$ group, respectively, in the dimensional reduction from $5+1$ to $4+1$ dimensions 9 ). 


\section{5. - A SIX-DIMENSIONAL PICTURE OF ELECTRIC-MAGNETIC DUALITY}

The five-dimensional picture of electric-magnetic duality in the (3+1)dimensional supersymmetry algebra given in Section 4 can be related to a six-dimensional one. The supersymmetry algebra for the supersymmetric SU(2) Yang-Mills theory in 5+l dimensions includes a surface integral 5),9)

$$
\sum_{\mu=0,1,2,3,5,6)}=\int_{(\mu)}^{5} d_{\mu}
$$

where $I_{o \mu}$ is the time-component of the antisymmetric tensor

$$
\begin{aligned}
& I_{\nu \mu}=\varepsilon_{\nu \mu \rho \sigma \tau \eta} F \rho \sigma a F \tau \eta a \\
& (\mu, \nu, \rho, \sigma, \tau, \eta=0,1,2,3,5,6 ; a=1,2,3)
\end{aligned}
$$

The sixth component of $Z_{\mu}$ gives rise, by dimensional reduction to $4+1$ dimensions, to the topological central charge $Z_{T}$ in (2.2) 9). The topological origin of $Z_{6}$ is given in Ref. 9). We wish to remind that there are some topological configurations which require periodicity on $x_{6}$ and the axial gauge $A_{6}=0$, to contribute at infinity to $\mathrm{Z}_{6}$.

The "vectorial magnetic charge" $G_{\mu}$ in (3.3) can also be obtained by dimensional reduction from $Z_{\mu}$, for $\mu \neq 6, A_{6}^{2} \equiv \phi^{2}$, in phase $\langle\phi\rangle \neq 0$.

Notice that both the instanton map and the Hopf map (4.13) can be obtained by dimensional reduction from the maps

$$
\Psi: S^{4} \longrightarrow S^{3}
$$

classified by the homotopy group

$$
\prod_{4}\left(S^{3}\right)=\mathbb{Z}_{2}
$$

where $S^{4}$ is the boundary of the five-dimensional Euclidean space $R_{E}^{5}$

$$
S^{4}=\left\{\left(x_{1}, x_{2}, x_{3}, x_{5}, x_{6}\right) \in R_{E}^{5} \mid \sum_{i} x_{i}^{2}=1\right\}
$$

The first case is illustrated in Ref. 9). Let us consider the second case. To the Hopf map (4.13), we can associate a suspended map : 


$$
3 h: X \in S^{4} \longrightarrow x \in S^{3}
$$

by mapping the $S^{3}$ equator of $S^{4}$ onto the $S^{2}$ equator of $S^{3}$ by $h$ and smoothly continuing to the poles. The Hopf map $h$ belongs to the element $l$ of $\pi_{3}\left(S^{2}\right)=\mathbb{Z}$. From this, one can deduce that the suspended Hopf map $\xi_{\text {h }}$ belongs to the non-trivial element of $\pi_{4}\left(S^{3}\right)=\mathbb{Z}_{2}$ [see Ref. 15), p. 312]. The same argument can be repeated for any map which belongs to an odd element of $\pi_{3}\left(S^{2}\right)$. Instead, maps belonging to an even element of $\pi_{3}\left(S^{2}\right)$ are mapped, by the suspension map $\xi$, into the trivial element of $\pi_{4}\left(S^{3}\right)$. The dimensional reduction from the suspended Hopf map (5.4) to the Hopf map (4.13) is given in the following commutative diagram of spheres :
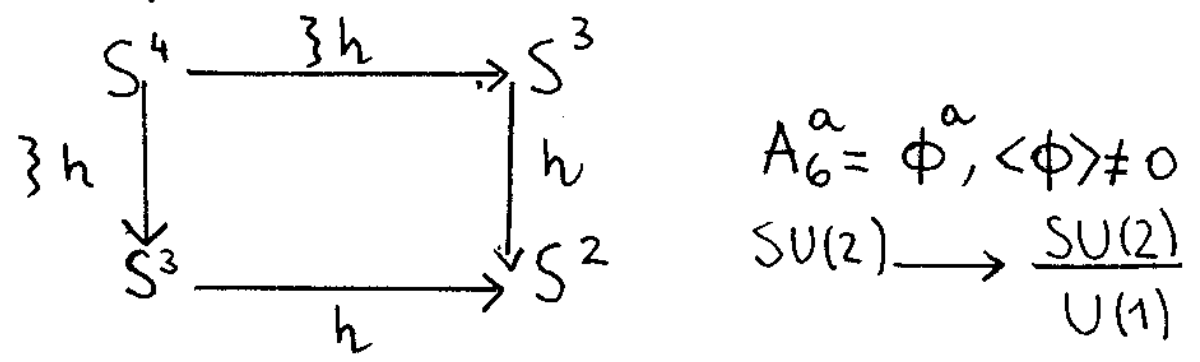

In the following scheme, we give a summary of the dimensional reduction from $5+1$ to $4+1$ dimensions (in both the phases of the theory) and from $4+1$ to $3+1$ dimensions, in terms of topological maps:

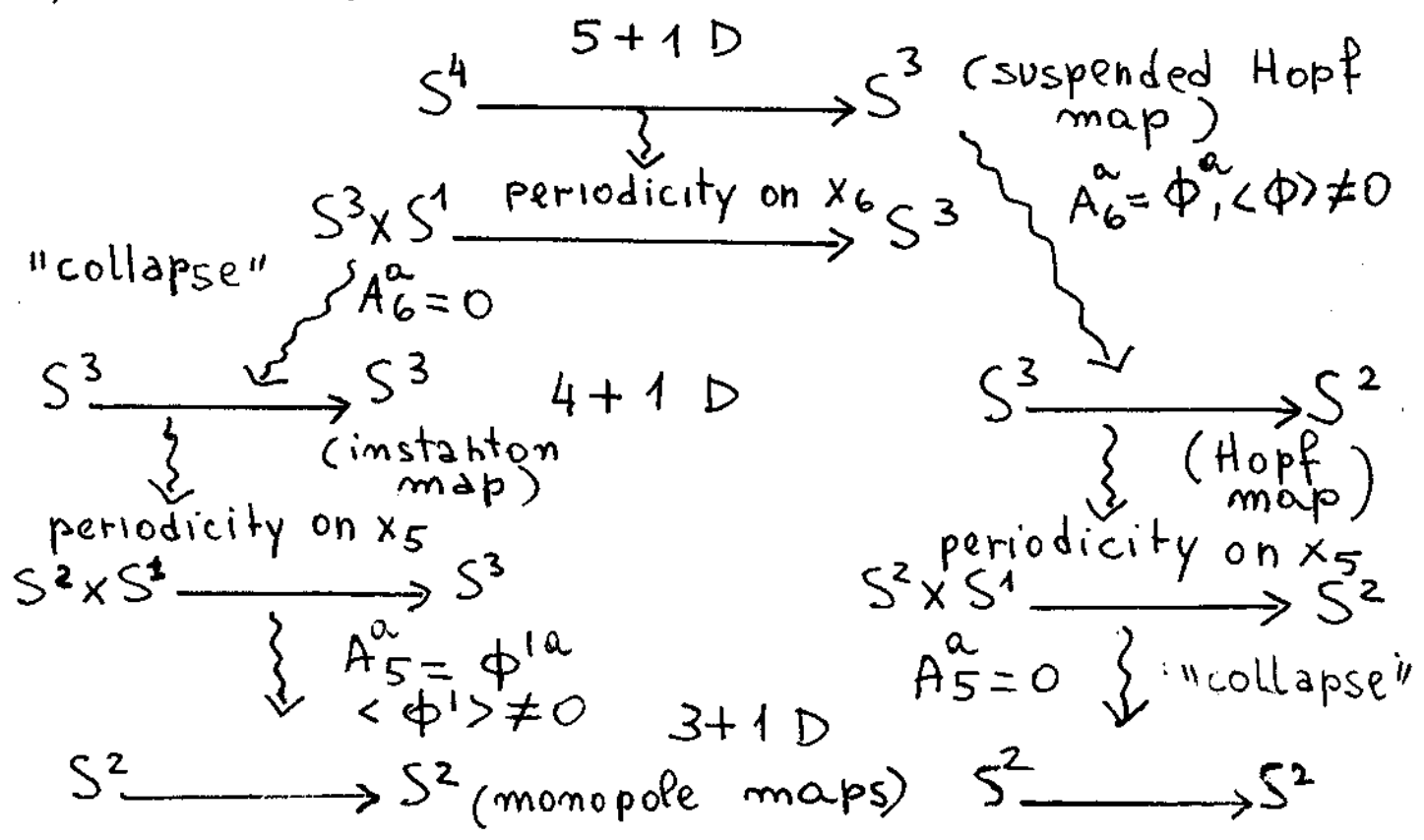


The details of the dimensional reduction of topological maps have been given in this paper for the right-hand side of scheme (5.6), while, for the left-hand side, they are given in Ref. 9).

The breaking of the Poincaré invariance of the supersymmetry algebra in the dimensional reduction from $5+1$ to $4+1$ dimensions (in both the phases of the theory) and from $4+1$ to $3+1$ dimensions is illustrated in summary by the following scheme:

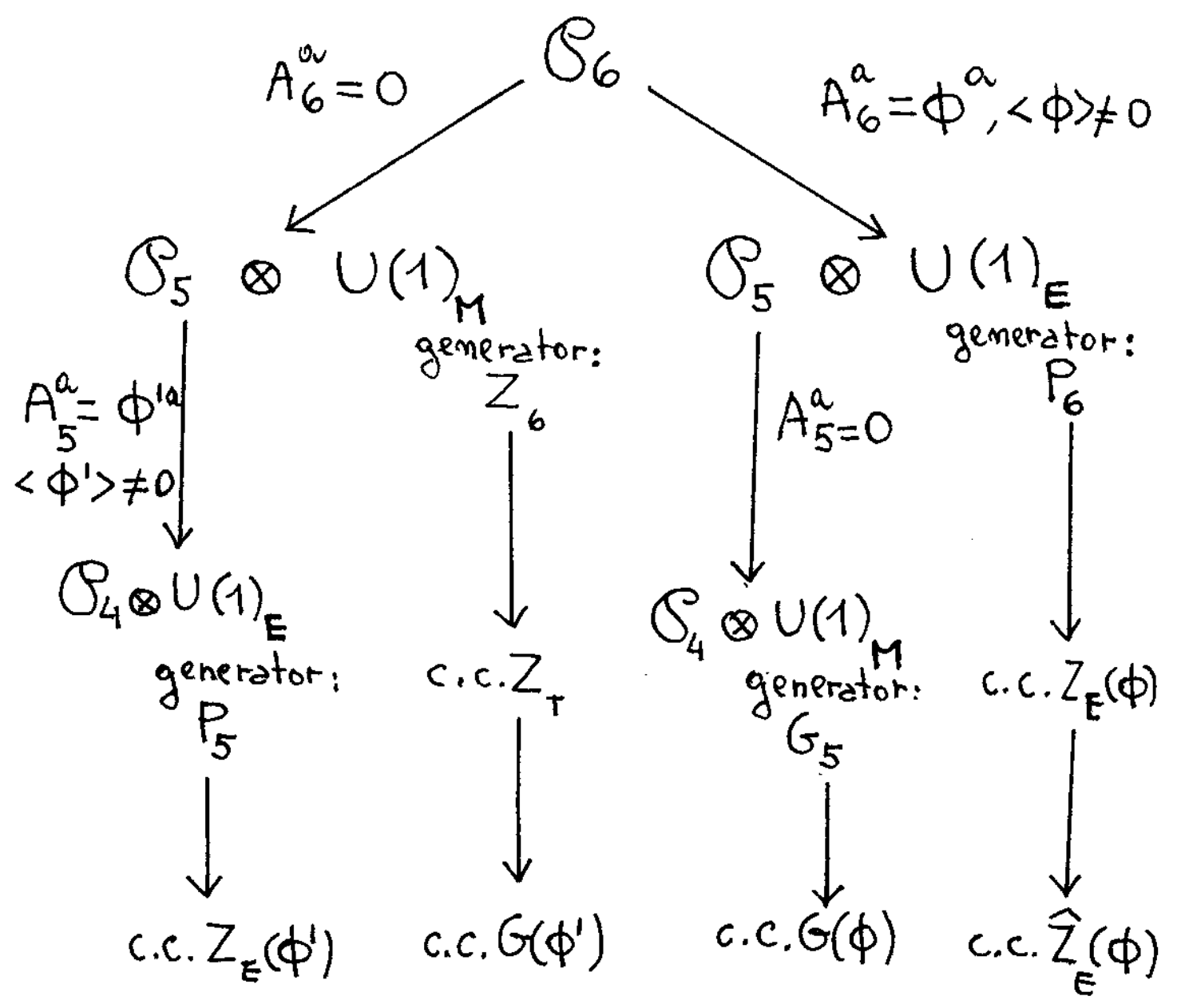

ACKNOWLEDGEMENTS

I have benefited from discussions on some topological arguments with L. Bonora, C.J. Isham, R. Percacci, P. Rossi and R. Stora. I also wish to thank D. Amati, S. Ferrara, T. Regge and J.G. Taylor for their interest in this work. 


\section{REFERENCES}

1) P. Goddard, J. Nuyts and D. Olive - Nuclear Phys. B125 (1977) 1.

2) C. Montonen and D. Olive - Phys.Letters 72B (1977) 117.

3) G. 't Hooft - Nuclear Phys. B79 (1974) 276 ;

A.M. Polyakov - JETP Letters 20 (1974) 194.

4) E. Witten and D. Olive - Phys.Letters 78B (1978) 97.

5) D. Olive - Nuclear Phys. B153 (1979) 1.

6) P.A. Zizzi - Nuclear Phys. B189 (1981) 317.

7) E. Cremmer and J. Scherk - Nuclear Phys. B118 (1977) 61.

8) T. Kaluza - Sitzungber.Preuss.Akad.Wiss.Phys.Math. Kl (1921) 866 ;

0. Klein - Z.Phys. 37 (1926) 895.

9) P.A. Zizzi - CERN Preprint TH. 3237 (1982), to be published in Phys.Rev.D.

10) T. Marinucci and P.A. Zizzi - Nuclear Phys. B195 (1982) 466.

11) N.E. Steenrod - Ann.Math. 48 (1947) 290.

12) H. Hopf - Math.Ann. 96 (1926) 209 ; ibid 96 (1926) 225 ; Commun.Math.Helv。 5 (1933) 39.

13) H.J. de Vega - Phys.Rev。D8 (1978) 2945.

14) G. Derrick - J.Math.Phys. 5 (1964) 1252.

15) S.T. Hu - Homotopy Theory, Academic Press (1959). 